Article

\title{
Cubic-Quartic Optical Soliton Perturbation with Differential Group Delay for the Lakshmanan-Porsezian-Daniel Model by Lie Symmetry
}

\author{
Sachin Kumar ${ }^{1}$ D, Anjan Biswas ${ }^{2,3,4,5,6}$, Yakup Yıldırım ${ }^{7}$, Luminita Moraru ${ }^{8, *} \mathbb{D}$, Simona Moldovanu ${ }^{9}$, \\ Hashim M. Alshehri ${ }^{3}$, Dalal Adnan Maturi ${ }^{3}$ and Dalal H. Al-Bogami ${ }^{3}$
}

check for updates

Citation: Kumar, S.; Biswas, A.; Yıldırım, Y.; Moraru, L.; Moldovanu, S.; Alshehri, H.M.; Maturi, D.A.; Al-Bogami, D.H. Cubic-Quartic Optical Soliton Perturbation with Differential Group Delay for the Lakshmanan-Porsezian-Daniel Model by Lie Symmetry. Symmetry 2022, 14, 224. https://doi.org/ $10.3390 /$ sym 14020224

Academic Editors: Calogero Vetro, Dariusz Wardowski and Bessem Samet

Received: 30 December 2021

Accepted: 21 January 2022

Published: 24 January 2022

Publisher's Note: MDPI stays neutral with regard to jurisdictional claims in published maps and institutional affiliations.

Copyright: () 2022 by the authors Licensee MDPI, Basel, Switzerland. This article is an open access article distributed under the terms and conditions of the Creative Commons Attribution (CC BY) license (https:/ / creativecommons.org/licenses/by/ $4.0 /)$.
1 Department of Mathematics and Statistics, Central University of Punjab, Bathinda 151401, Punjab, India; sachiljan@gmail.com

2 Department of Applied Mathematics, National Research Nuclear University, 31 Kashirskoe Hwy, 115409 Moscow, Russia; biswas.anjan@gmail.com

3 Mathematical Modeling and Applied Computation (MMAC) Research Group, Department of Mathematics, King Abdulaziz University, Jeddah 21589, Saudi Arabia; hmalshehri@kau.edu.sa (H.M.A.); dmaturi@kau.edu.sa (D.A.M.); gno_18@hotmail.com (D.H.A.-B.)

4 Department of Applied Sciences, Cross-Border Faculty, Dunarea de Jos University of Galati, 111 Domneasca Street, 800201 Galati, Romania

5 Department of Mathematics and Applied Mathematics, Sefako Makgatho Health Sciences University, Medunsa 0204, South Africa

6 Department of Physics, Chemistry and Mathematics, Alabama A\&M University, Normal, AL 35762-4900, USA

7 Department of Mathematics, Faculty of Arts and Sciences, Near East University, Nicosia 99138, Cyprus; yakup.yildirim@neu.edu.tr

8 Department of Chemistry, Physics and Environment, Faculty of Sciences and Environment, Dunarea de Jos University of Galati, 47 Domneasca Street, 800008 Galati, Romania

9 Department of Computer Science and Information Technology, Faculty of Automation, Computers, Electrical Engineering and Electronics, Dunarea de Jos University of Galati, 47 Domneasca Street, 800008 Galati, Romania; simona.moldovanu@ugal.ro

* Correspondence: luminita.moraru@ugal.ro

Abstract: This paper employs Lie symmetry analysis to recover cubic-quartic optical soliton solutions to the Lakshmanan-Porsezian-Daniel model in birefringent fibers. The results are a sequel to the previously reported work on the same model in unpolarized fibers. Dark, singular, and straddled optical solitons that emerged from the scheme are presented.

Keywords: solitons; birefringence; Lakshmanan-Porsezian-Daniel equation; Lie symmetry

\section{Introduction}

The concept of cubic-quartic (CQ) optical solitons emerged a couple of years ago out of extreme necessity when the chromatic dispersion (CD) effect ran low. This led to replenishing the low $C D$ count with the $C Q$ dispersive effect so that the necessary balance between CD and self-phase modulation (SPF) was sustained for the existence and propel of solitons for long distances through optical fibers. Later, this concept was applied to the Lakshmanan-Porsezian-Daniel (LPD) model [1-4], not including the well-known and most visible one: the nonlinear Schrödinger's equation. The vector-coupled LPD equation in birefringent fibers was considered with the aid of the extended version of Jacobi's elliptic function expansion scheme in [1], where Jacobi's doubly periodic wave solutions are found. These solutions, in the limiting case, give rise to dark solitons, singular solitons or periodic solutions. The generalized LPD model with arbitrary refractive index is considered via the Jacobi and Weierstrass elliptic functions in [2], where solitary waves corresponding to optical solitons are recovered for an arbitrary refractive index. Periodic waves are also revealed for the classical case at $n=1$ and for the new case $n=1 / 2$. The 
LPD model is investigated, with the aid of the Ablowitz-Kaup-Newell-Segur (AKNS) hierarchy, to discover novel soliton breathers on the zero background in [3], where the specific conditions for the appearance of the standing and moving soliton breathers are also derived. Additionally, simple formulas for the soliton-breather periods are structured to understand the possibilities for controlling breather dynamics. The LPD equation in polarization-preserving fibers is discussed by virtue of the complete discrimination system for the polynomial method to obtain a complete list of all envelope patterns and to show a variety of dynamical properties of the patterns in [4], where solitons, singularity, periodicity, and double periodicity emerged from the exact parameter condition of existence for each pattern. Several results have been recovered using the CQ-LPD model with its scalar version [5-7]. The CQ-LPD equation is adopted using the Lie symmetry analysis in [5], where straddled singular-bright optical solitons are reported using the reduced ordinary differential equations that are handled using Kudryashov's method. The perturbed CQ-LPD model is addressed by the method of undetermined coefficients in [6], where polarization-preserving fibers and birefringent fibers are studied. The conservation laws for polarization-preserving fibers are also retrieved and enumerated. The existence criteria for the displayed solitons are also presented. The perturbed CQ-LPD model is employed for both with and without polarization in [7], where a full spectrum of soliton solutions are retrieved using the sine-Gordon equation approach. Powerful Lie symmetry analysis has been successfully applied to several models that arise in mathematical physics $[1-4,6-13]$. In particular, a successful application of Lie symmetry analysis to the scalar version of the CQ-LPD model, yielded dark and singular solitons [5]. Lie symmetry is one of the most powerful methods for obtaining optical solitons with the governing models in nonlinear optics. In the last few decades, Lie's method has been described in a number of excellent textbooks and has been applied to a number of physical and engineering models. The study of the group of infinitesimal transformations, in other words, Lie group point transformations, has an important place in this method. The Lie symmetry method, developed in the 19th century (1842-1899) by the Norwegian mathematician Sophus Lie, is exceptionally algorithmic. This method systematically combines and expands famous ad hoc methodologies for constructing optical solitons with model equations in optical fiber communications.

Turning the page, and moving forward, it is about time to address the CQ-LPD model in birefringent fibers when polarization-mode dispersion kicks in. The perturbation terms are also taken into account in the vector CQ-LPD model. Lie symmetry analysis, when applied, leads to coupled ordinary differential equations (ODEs) [14,15]. These ODEs would be subsequently addressed using two powerful integration schemes: Kudryashov's method and the improved F-expansion scheme. These lead to dark and singular soliton solutions to the model. The details of Lie symmetry analysis followed by the derivation of the soliton solutions from the derived ODEs are detailed and exhibited in the rest of the paper after a succinct introduction to the model.

\section{Governing Model}

The perturbed LPD equation for propagation of CQ solitons through birefringent fibers is given in its dimensionless form [6,7]:

$$
\begin{gathered}
i q_{t}+i a_{1} q_{x x x}+b_{1} q_{x x x x}+\left(c_{1}|q|^{2}+d_{1}|r|^{2}\right) q=\left(\alpha_{1}\left(q_{x}\right)^{2}+\beta_{1}\left(r_{x}\right)^{2}\right) q^{*} \\
+\left(\gamma_{1}\left|q_{x}\right|^{2}+\lambda_{1}\left|r_{x}\right|^{2}\right) q+\left(\delta_{1}|q|^{2}+\zeta_{1}|r|^{2}\right) q_{x x}+\left(\mu_{1} q^{2}+\rho_{1} r^{2}\right) q_{x x}^{*} \\
+\left(f_{1}|q|^{4}+g_{1}|q|^{2}|r|^{2}+h_{1}|r|^{4}\right) q+i\left[\eta_{1}\left(|q|^{2} q\right)_{x}+v_{1}\left(|r|^{2} r\right)_{x}\right. \\
\left.+\left\{\theta_{1}\left(|q|^{2}\right)_{x}+\epsilon_{1}\left(|r|^{2}\right)_{x}\right\} q+\left(\tau_{1}|q|^{2}+\sigma_{1}|r|^{2}\right) q_{x}\right],
\end{gathered}
$$

and

$$
\begin{gathered}
i r_{t}+i a_{2} r_{x x x}+b_{2} r_{x x x x}+\left(c_{2}|r|^{2}+d_{2}|q|^{2}\right) r=\left(\alpha_{2}\left(r_{x}\right)^{2}+\beta_{2}\left(q_{x}\right)^{2}\right) r^{*} \\
+\left(\gamma_{2}\left|r_{x}\right|^{2}+\lambda_{2}\left|q_{x}\right|^{2}\right) r+\left(\delta_{2}|r|^{2}+\zeta_{2}|q|^{2}\right) r_{x x}+\left(\mu_{2} r^{2}+\rho_{2} q^{2}\right) r_{x x}^{*} \\
+\left(f_{2}|r|^{4}+g_{2}|q|^{2}|r|^{2}+h_{2}|q|^{4}\right) r+i\left[\eta_{2}\left(|r|^{2} r\right)_{x}+v_{2}\left(|q|^{2} q\right)_{x}\right. \\
\left.+\left\{\theta_{2}\left(|r|^{2}\right)_{x}+\epsilon_{2}\left(|q|^{2}\right)_{x}\right\} r+\left(\tau_{2}|r|^{2}+\sigma_{2}|q|^{2}\right) r_{x}\right]
\end{gathered}
$$


where the complex-valued functions $q=q(x, t)$ and $r=r(x, t)$ represent wave profiles in birefringent fibers with the complex parameter $i$ representing $\sqrt{-1}$. The parameters $a_{l}$ and $b_{l}$ for $l=(1,2)$, are the coefficients of third-order and fourth-order dispersions, respectively. Next, $c_{l}$ and $f_{l}$ are the coefficients of self-phase modulation, while $h_{l}, g_{l}$, and $d_{l}$ indicate the effects of cross-phase modulation. The parameters $\eta_{l}$ and $v_{l}$ denote self steepening. Then, $\tau_{l}, \epsilon_{l}, \theta_{l}, v_{l}, \eta_{l}, \rho_{l}, \mu_{l}$, and $\sigma_{l}$ stem from nonlinear dispersive effects along with the two components of birefringence. Finally, $\alpha_{l}, \beta_{l}, \gamma_{l}$, and $\lambda_{l}$ are additional nonlinear effects.

\section{Lie Symmetry Analysis}

The Lie symmetry method [5,14-17] is implemented in the coupled CQ-LPD model with perturbation terms for birefringent fibers (1) and (2) in this section. To solve model (1) and (2), the wave transformations are structured as follows:

$$
\begin{aligned}
& q(x, t)=u(x, t) e^{i v(x, t),} \\
& r(x, t)=w(x, t) e^{i v(x, t),}
\end{aligned}
$$

where the real-valued functions $u=u(x, t)$ and $w=w(x, t)$ are the amplitude components of the soliton, while the real-valued function $v=v(x, t)$ is the phase component of the soliton. Inserting Equation (3) into Equation (1), we have the real and imaginary parts:

$$
\begin{gathered}
\left(\frac{\partial^{4} u}{\partial x^{4}}\right) b_{1}+\left\{-u a_{1}-4\left(\frac{\partial v}{\partial x}\right) u b_{1}\right\} \frac{\partial^{3} v}{\partial x^{3}}-3 u\left(\frac{\partial^{2} v}{\partial x^{2}}\right)^{2} b_{1}+\left\{-12\left(\frac{\partial u}{\partial x}\right) b_{1} \frac{\partial v}{\partial x}\right. \\
\left.-3\left(\frac{\partial u}{\partial x}\right) a_{1}\right\} \frac{\partial^{2} v}{\partial x^{2}}+\left\{-6\left(\frac{\partial v}{\partial x}\right)^{2} b_{1}-3\left(\frac{\partial v}{\partial x}\right) a_{1}-3\left(\frac{\mu_{1}}{3}+\frac{\delta_{1}}{3}\right) u^{2}\right. \\
\left.-w^{2}\left(\rho_{1}+\zeta_{1}\right)\right\} \frac{\partial^{2} u}{\partial x^{2}}+\left(\frac{\partial v}{\partial x}\right) u b_{1}+\left(\frac{\partial v}{\partial x}\right)^{3} u a_{1}+\left\{\left(\mu_{1}+\alpha_{1}+\delta_{1}-\gamma_{1}\right) u^{2}\right. \\
\left.+\left(\rho_{1}+\beta_{1}+\zeta_{1}-\lambda_{1}\right) w^{2}\right\} u\left(\frac{\partial v}{\partial x}\right)^{2}+\left\{\left(\eta_{1}+\tau_{1}\right) u^{3}+w^{2} u \sigma_{1}+w^{3} s_{1}\right\} \frac{\partial v}{\partial x} \\
-u\left(\alpha_{1}+\gamma_{1}\right)\left(\frac{\partial u}{\partial x}\right)^{2}-u\left(\beta_{1}+\lambda_{1}\right)\left(\frac{\partial w}{\partial x}\right)^{2}-u \frac{\partial v}{\partial t} \\
-u^{5} f_{1}+\left\{-w^{2} g_{1}+c_{1}\right\} u^{3}+\left\{-w^{4} h_{1}+d_{1} w^{2}\right\} u=0,
\end{gathered}
$$

and

$$
\begin{gathered}
-3 w\left(w s_{1}+\frac{2}{3} u \epsilon_{1}\right) \frac{\partial w}{\partial x}+4\left(\frac{\partial u}{\partial x}\right) b_{1} \frac{\partial^{3} v}{\partial x^{3}}+\left(a_{1}+4\left(\frac{\partial v}{\partial x}\right) b_{1}\right) \frac{\partial^{3} u}{\partial x^{3}}+\left[6\left(\frac{\partial^{2} u}{\partial x^{2}}\right) b_{1}\right. \\
\left.-6\left(\frac{\partial v}{\partial x}\right)^{2} u b_{1}-3 u a_{1} \frac{\partial v}{\partial x}+u\left\{\left(\mu_{1}-\delta_{1}\right) u^{2}+w^{2}\left(\rho_{1}-\zeta_{1}\right)\right\}\right] \frac{\partial^{2} v}{\partial x^{2}}-4\left(\frac{\partial u}{\partial x}\right) b_{1}\left(\frac{\partial v}{\partial x}\right)^{3} \\
-3\left(\frac{\partial u}{\partial x}\right) a_{1}\left(\frac{\partial v}{\partial x}\right)^{2}+\left[-2\left\{\left(\delta_{1}+\alpha_{1}-\mu_{1}\right) u^{2}-\frac{2}{3}\left(\rho_{1}-\zeta_{1}\right) w^{2}\right\} \frac{\partial u}{\partial x}-2 w\left(\frac{\partial w}{\partial x}\right) u \beta_{1}\right] \frac{\partial v}{\partial x} \\
+\left\{-\left(3 \eta_{1}+\tau_{1}+2 \theta_{1}\right) u^{2}-w^{2} \sigma_{1}\right\} \frac{\partial u}{\partial x}+\left(\frac{\partial^{4} v}{\partial x^{4}}\right) u b_{1}+\frac{\partial u}{\partial t}=0 .
\end{gathered}
$$

Similarly, inserting Equation (3) into Equation (2), we have the real and imaginary portions, which due to lengthy expressions, we have not written here. For the above system of equations, let us consider a one-parameter $(\epsilon)$ Lie group of transformations as follows:

$$
\begin{gathered}
x^{*}=x+\epsilon \xi(x, t, u, v, w)+O\left(\epsilon^{2}\right), \\
t^{*}=t+\epsilon \tau(x, t, u, v, w)+O\left(\epsilon^{2}\right), \\
u^{*}=u+\epsilon \eta_{1}(x, t, u, v, w)+O\left(\epsilon^{2}\right), \\
v^{*}=v+\epsilon \eta_{2}(x, t, u, v, w)+O\left(\epsilon^{2}\right), \\
w^{*}=v+\epsilon \eta_{3}(x, t, u, v, w)+O\left(\epsilon^{2}\right),
\end{gathered}
$$

where $\xi, \tau$ and $\eta_{l}$ for $l=1,2,3$ are infinitesimals and $\epsilon<<1$ is a very small parameter. The associated vector field for Equation (6) is indicated below:

$$
V=\xi \partial_{x}+\tau \partial_{t}+\eta_{1} \partial_{u}+\eta_{2} \partial_{v}+\eta_{3} \partial_{w} .
$$


Hence, the fourth prolongation formula [14,15] for Equation (7) is considered as

$$
\begin{gathered}
p r^{(4)} V=V+\eta_{1}^{t} \frac{\partial}{\partial u_{t}}+\eta_{2}^{t} \frac{\partial}{\partial v_{t}}+\eta_{3}^{t} \frac{\partial}{\partial w_{t}}+\eta_{1}^{x} \frac{\partial}{\partial u_{x}}+\eta_{2}^{x} \frac{\partial}{\partial v_{x}}+\eta_{3}^{x} \frac{\partial}{\partial w_{x}}+\eta_{1}^{x x x} \frac{\partial}{\partial u_{x x x}} \\
+\eta_{2}^{x x x} \frac{\partial}{\partial v_{x x x}}+\eta_{3}^{x x x} \frac{\partial}{\partial w_{x x x}}+\eta_{1}^{x x x x} \frac{\partial}{\partial u_{x x x x}}+\eta_{2}^{x x x x} \frac{\partial}{\partial v_{x x x x}}+\eta_{3}^{x x x x} \frac{\partial}{\partial w_{x x x x}}
\end{gathered}
$$

where $\eta_{1}^{t}, \eta_{2}^{t}, \eta_{3}^{t}, \eta_{1}^{x}, \eta_{2}^{x}, \eta_{3}^{x}, \eta_{1}^{x x x}, \eta_{2}^{x x x}, \eta_{3}^{x x x}, \eta_{1}^{x x x x}, \eta_{2}^{x x x x}$, and $\eta_{3}^{x x x x}$ represent the extended infinitesimals. By using $\mathrm{pr}^{(4)} V(\Delta)=0$ whenever $\Delta=0$ in Equations (4) and (5), and other equations obtained from Equation (2), we obtain the invariance conditions. Plugging the extended infinitesimals into the invariance condition and equating the coefficients of different derivative terms that are equal to zero leads to the following:

$$
\xi=C_{2}, \tau=C_{1}, \eta_{1}=0, \eta_{2}=C_{3}, \eta_{3}=0,
$$

where $C_{l}, l=1,2,3$ are arbitrary constants. Thus, we obtain only trivial symmetries.

\subsection{Symmetry Reduction and Closed-Form Solutions}

The transformations are employed to reduce the governing Equation (4) to the system of ODEs in this section. The corresponding characteristic equation is indicated below

$$
\frac{d x}{\xi(x, t, u, v, w)}=\frac{d t}{\tau(x, t, u, v, w)}=\frac{d u}{\eta_{1}(x, t, u, v, w)}=\frac{d v}{\eta_{2}(x, t, u, v, w)}=\frac{d w}{\eta_{3}(x, t, u, v, w)},
$$

where $\xi, \tau, \eta_{1}, \eta_{2}$, and $\eta_{3}$ are given by Equation (9). Let us consider the following values of infinitesimals:

$$
\xi=k_{1}, \tau=1, \eta_{1}=0, \eta_{2}=k_{2}, \eta_{3}=0,
$$

where $k_{1}$ and $k_{2}$ are arbitrary real numbers. Now, corresponding to Equation (11), on solving Equation (10), the similarity variables are structured as the following:

$$
\begin{gathered}
\sigma=x-k_{1} t, \\
u=F(\sigma), \\
v=k_{2} t+G(\sigma), \\
w=H(\sigma),
\end{gathered}
$$

along with the dependent variables $F$ and $G$. For simplification, let us take the following:

$$
F(\sigma)=H(\sigma),
$$

and on substituting Equation (12) into Equations (4) and (5) and the remaining system of equations, we obtain the following system of equations:

$$
\begin{gathered}
\left(-\beta_{l}-\alpha_{l}-\gamma_{l}-\lambda_{l}\right) F\left(F^{\prime}\right)^{2}-\left(12 b_{l} G^{\prime} G^{\prime \prime}+3 a_{l} G^{\prime \prime}\right) F^{\prime}-\left\{\left(\mu_{l}+\rho_{l}+\zeta_{l}+\delta_{l}\right) F^{2}\right. \\
\left.+6 b_{l}\left(G^{\prime}\right)^{2}+3 a_{l} G^{\prime}\right\} F^{\prime \prime}+\left(-g_{l}-h_{l}-f_{l}\right) F^{5}+\left\{\left(\mu_{1}+\rho_{l}+\beta_{l}+\zeta_{l}+\delta_{l}+\alpha_{l}\right.\right. \\
\left.\left.-\gamma_{l}-\lambda_{l}\right)\left(G^{\prime}\right)^{2}+\left(s_{l}+\eta_{l}+\sigma_{l}+\tau_{l}\right) G^{\prime}+d_{l}+c_{l}\right\} F^{3}+\left\{a_{l}\left(G^{\prime}\right)^{3}+b_{l}\left(G^{\prime}\right)^{4}\right. \\
\left.+\left(-4 b_{l} G^{\prime \prime \prime}+k_{1}\right) G^{\prime}-3 b_{l}\left(G^{\prime \prime}\right)^{2}-a_{l} G^{\prime \prime \prime}-k_{2}\right\} F+b_{l} F^{\prime \prime \prime \prime}=0,
\end{gathered}
$$

and

$$
\begin{gathered}
\left(-2 \epsilon_{l}-3 s_{l}+\left(-2 \alpha_{l}-2 \delta_{l}+2 \rho_{l}+2 \mu_{l}-2 \beta_{l}-2 \zeta_{l}\right) G^{\prime}-\tau_{l}-3 \eta_{l}-\sigma_{l}-2 \theta_{l}\right) F^{2} F^{\prime} \\
+6 b_{l} F^{\prime \prime} G^{\prime \prime}+\left(4 b_{l} G^{\prime \prime \prime}-4 b_{l} G^{\prime 3}-3 a_{l} G^{\prime 2}-k_{1}\right) F^{\prime}+\left(\mu_{l}-\delta_{l}+\rho_{l}-\zeta_{l}\right) G^{\prime \prime} F^{3} \\
+\left(a_{l}+4 b_{l} G^{\prime}\right) F^{\prime \prime \prime}+\left\{\left(-6 b_{l}\left(G^{\prime}\right)^{2}-3 a_{l} G^{\prime}\right) G^{\prime \prime}+b_{l} G^{\prime \prime \prime \prime}\right\} F=0,
\end{gathered}
$$

where $\left({ }^{\prime}\right)$ indicates the derivative with respect to $\sigma$ and $l=1,2$. In Equation (15), setting

$$
4 b_{l} G^{\prime}+a_{l}=0,
$$

we have

$$
G(\sigma)=-\frac{a_{l}}{4 b_{l}} \sigma+C_{1},
$$

where $C_{1}$ is arbitrary constant. In addition, with Equation (17), by setting the coefficients of $F^{\prime}$ equal to zero in Equation (15), we have the following: 


$$
k_{1}=-\frac{a_{l}^{3}}{8 b_{l}^{2}}
$$

Equations (14) and (15) reduce to

$$
\begin{gathered}
-\left(g_{l}+f_{l}+h_{l}\right) F^{5}+\frac{3 a_{1}{ }^{2}}{8 b_{1}} F^{\prime \prime}+b_{1} F^{\prime \prime \prime \prime} \\
+\frac{4\left(c_{l}+d_{l}\right) b_{1}{ }^{2}+2 a_{1}\left(\eta_{l}+\theta_{l}+\epsilon_{l}+s_{l}\right) b_{1}-a_{1}^{2}\left(\zeta_{l}+\delta_{l}\right)}{4 b_{1}{ }^{2}} F^{3}=0,
\end{gathered}
$$

with the following parameter constraints:

$$
\begin{gathered}
\mu_{l}=-\zeta_{l}-\rho_{l}-\delta_{l} \\
\lambda_{l}=2 \delta_{l}+2 \zeta_{l}-\gamma_{l}-\frac{2 b_{1}\left(2 \epsilon_{l}+2 \theta_{l}+3 s_{l}+3 \eta_{l}+\sigma_{l}+\tau_{l}\right)}{a_{1}} \\
\alpha_{l}=-3 s_{l}-2 \epsilon_{l}-\sigma_{l}-3 \eta_{l}-\tau_{l}-2 \theta_{l}-\frac{\left(2 \rho_{l}-2 \beta_{l}-2 \zeta_{l}-2 \delta_{l}-2 \alpha_{l}+2 \mu_{l}\right) a_{1}}{4 b_{1}} .
\end{gathered}
$$

\subsubsection{The Generalized Kudryashov's Method}

We obtain CQ optical solitons with the governing system (1) and (2) by solving the ODE (19) for $l=1,2$. Equation (19) admits the solution form $[2,12]$ :

$$
F(\sigma)=\frac{\sum_{i=0}^{N} m_{i} R^{i}(\sigma)}{\sum_{i=0}^{M} n_{i} R^{j}(\sigma)}
$$

where $m_{i}(i=0,1,2, \ldots, N)$ and $n_{j}(j=0,1,2, \ldots, M)$ are real-valued constants. In addition, the new function $R=R(\sigma)$ satisfies the following ODE:

$$
R^{\prime}(\sigma)=R^{2}(\sigma)-R(\sigma),
$$

along with the solution

$$
R(\sigma)=\frac{1}{1+A e^{\sigma}}
$$

where $A$ is arbitrary constant. By the usage of balance principle in Equation (19), we obtain $N=M+1$. By taking $M=1$, we have $N=2$. So, Equation (21) becomes

$$
F(\sigma)=\frac{m_{0}+m_{1} R(\sigma)+m_{2} R^{2}(\sigma)}{n_{0}+n_{1} R(\sigma)}
$$

where $m_{0}, m_{1}, m_{2}, n_{0}$, and $n_{1}$ are real-valued constants. Inserting Equation (24), along with Equation (22), into Equation (19), we arrive at the following results:

Set-I:

$$
\begin{aligned}
m_{0}=0, m_{1} & =-m_{2}, n_{1}=0, f_{l}=-g_{l}-h_{l}, a_{l}= \pm \frac{2 \sqrt{30} i}{3} b_{l}, k_{2}=-\frac{19 a_{l}}{36}, \\
c_{l} & =\mp \frac{i m_{2}^{2}\left(\epsilon_{l}+\eta_{l}+v_{l}+\theta_{l}\right) \sqrt{30}+\left(3 d_{l}+10 \zeta_{l}+10 \delta_{l}\right) m_{2}{ }^{2}+360 n_{0}^{2} a_{l}}{3 m_{2}{ }^{2}},
\end{aligned}
$$

where $m_{2}$ and $n_{0}$ are arbitrary constants. Therefore, CQ straddled optical solitons with Equation (1) are structured as

$$
q(x, t)=-\frac{m_{2} A\left\{\cosh \left(t k_{1}-x\right)-\sinh \left(t k_{1}-x\right)\right\}}{n_{0}\left[1+A\left\{\cosh \left(t k_{1}-x\right)-\sinh \left(t k_{1}-x\right)\right\}\right]^{2}} e^{i\left\{\left(k_{2}+\frac{a_{1}}{4 b_{1}} k_{1}\right) t-\frac{a_{1}}{4 b_{1}} x+C_{1}\right\}},
$$

and

$$
r(x, t)=-\frac{m_{2} A\left\{\cosh \left(t k_{1}-x\right)-\sinh \left(t k_{1}-x\right)\right\}}{n_{0}\left[1+A\left\{\cosh \left(t k_{1}-x\right)-\sinh \left(t k_{1}-x\right)\right\}\right]^{2}} e^{i\left\{\left(k_{2}+\frac{a_{1}}{4 b_{1}} k_{1}\right) t-\frac{a_{1}}{4 b_{1}} x+C_{1}\right\}} .
$$

The CQ straddled optical solitons (26) and (27) are given with the conditions (18), (20), and (25) by taking $l=1$. CQ straddled optical solitons with Equation (2) are also yielded by taking $l=2$.

Set-II:

$$
\begin{gathered}
m_{0}=-\frac{m_{1}}{2}, m_{2}=0, n_{1}=0, a_{l}= \pm \frac{4 \sqrt{3} b_{l}}{3}, \\
f_{l}=-\frac{-24 n_{0}{ }^{4} b_{l}+m_{1}{ }^{4} g_{l}+m_{1}{ }^{4} h_{l}}{m_{1}{ }^{4}}, k_{2}=\frac{5 a_{l}^{4}}{256 b_{l}^{3}} \\
c_{l}=\mp \frac{\left(\begin{array}{c}
2 \sqrt{3} m_{1}{ }^{2} \epsilon_{l}+2 \sqrt{3} m_{1}{ }^{2} \eta_{l}+2 \sqrt{3} m_{1}{ }^{2} v_{1}+2 \sqrt{3} m_{1}{ }^{2} \theta_{l} \\
+3 m_{1}{ }^{2} d_{l}-4 m_{1}{ }^{2} \delta_{l}-4 m_{1}{ }^{2} \zeta_{l}-18 n_{0}{ }^{2} b_{l}
\end{array}\right)}{3 m_{1}{ }^{2}},
\end{gathered}
$$


where $m_{1}$ and $n_{0}$ are arbitrary constants. Thus, CQ straddled optical solitons with Equation (1) are indicated below:

$$
q(x, t)=-\frac{m_{1}\left[A\left\{\cosh \left(k_{1} t-x\right)-\sinh \left(k_{1} t-x\right)\right\}-1\right]}{2 n_{0}\left[1+A\left\{\cosh \left(k_{1} t-x\right)-\sinh \left(k_{1} t-x\right)\right\}\right]} e^{i\left\{\left(k_{2}+\frac{a_{1}}{4 b_{1}} k_{1}\right) t-\frac{a_{1}}{4 b_{1}} x+C_{1}\right\}},
$$

and

$$
r(x, t)=-\frac{m_{1}\left[A\left\{\cosh \left(k_{1} t-x\right)-\sinh \left(k_{1} t-x\right)\right\}-1\right]}{2 n_{0}\left[1+A\left\{\cosh \left(k_{1} t-x\right)-\sinh \left(k_{1} t-x\right)\right\}\right]} e^{i\left\{\left(k_{2}+\frac{a_{1}}{4 b_{1}} k_{1}\right) t-\frac{a_{1}}{4 b_{1}} x+C_{1}\right\}} .
$$

The CQ straddled optical solitons are retrieved by virtue of the parameter constraints (18), (20), and (28) by taking $l=1$. CQ straddled optical solitons to Equation (2) are also revealed by taking $l=2$. Equations (29) and (30) represent optical vector solitons that are constituted by the two polarization components of the optical field.

\subsubsection{Improved F-Expansion Approach}

New solitary wave solutions with Equation (1) are reported by solving the ODE (19) for $l=1,2$ Equation (19) presumes the formal solution [5]

$$
F(\sigma)=\sum_{i=-N}^{N} A_{i}(b+H(\sigma))^{i},
$$

where the new function $H(\sigma)$ satisfies the ODE

$$
H^{\prime}(\sigma)=z_{0}+z_{1} H(\sigma)+z_{2} H(\sigma)^{2}+z_{3} H(\sigma)^{3}
$$

where $z_{0}, z_{1}, z_{2}, z_{3}$, and $A_{i}$ are arbitrary constants. By the use of balance principle in Equation (19), we obtain $N=1$. So, Equation (31) reduces to

$$
F(\sigma)=A_{-1}(b+H(\sigma))^{-1}+A_{0}+A_{1}(b+H(\sigma)) .
$$

Plugging Equation (33), along with Equation (32), into Equation (19), we arrive at the following results:

Family-1: $\left(z_{2}=z_{3}=0\right)$

Set-I:

$$
\begin{gathered}
z_{0}=z_{1} b, A_{0}=0, A_{1}=0, f_{l}=-g_{l}-h_{l}, a_{l}=\frac{2 i \sqrt{6} z_{1} b_{l}}{3}, \\
c_{l}= \pm \frac{i \sqrt{6} z_{1}}{3}\left(\eta_{l}+\epsilon_{l}+\theta_{l}+v_{l}\right)-\frac{2 z_{1}^{2}}{3}\left(\zeta_{l}+\delta_{l}\right)-d_{l} .
\end{gathered}
$$

Hence, the analytical solutions of Equation (1) are formulated as

$$
q(x, t)=\frac{A_{-1}}{e^{z_{1}\left(k_{1} t-x\right)}} e^{i\left\{\left(k_{2}+\frac{a_{1}}{4 b_{1}} k_{1}\right) t-\frac{a_{1}}{4 b_{1}} x+C_{1}\right\}},
$$

and

$$
r(x, t)=\frac{A_{-1}}{e^{z_{1}\left(k_{1} t-x\right)}} e^{i\left\{\left(k_{2}+\frac{a_{1}}{4 b_{1}} k_{1}\right) t-\frac{a_{1}}{4 b_{1}} x+C_{1}\right\}} .
$$

The explicit solutions (35) and (36) are derived from the constraints (18), (20), and (34) by taking $l=1$. The exact solutions of Equation (2) are also obtained by taking $l=2$.

Set-II:

$$
\begin{gathered}
A_{-1}=0, A_{0}=A_{1}=\text { arbitrary, } f_{l}=-g_{l}-h_{l}, a_{l}=\frac{2}{3} i \sqrt{6} b_{l} z_{1}, \\
c_{l}=-\frac{z_{1} i}{3}\left(\epsilon_{l}+\theta_{l}+v_{l}+\eta_{l}\right) \sqrt{6}-\frac{2 z_{1}^{2}}{3}\left(\delta_{l}+\zeta_{l}\right)-d_{l} .
\end{gathered}
$$

Consequently, the explicit solutions of Equation (1) are considered as

$$
q(x, t)=\left(A_{1} e^{z_{1}\left(k_{1} t-x\right)} C_{2}+A_{1} b+A_{0}-\frac{A_{1} z_{0}}{z_{1}}\right) e^{i\left\{\left(k_{2}+\frac{a_{1}}{4 b_{1}} k_{1}\right) t-\frac{a_{1}}{4 b_{1}} x+C_{1}\right\}},
$$

and

$$
r(x, t)=\left(A_{1} e^{z_{1}\left(k_{1} t-x\right)} C_{2}+A_{1} b+A_{0}-\frac{A_{1} z_{0}}{z_{1}}\right) e^{i\left(k_{2} t-\frac{a_{2}}{4 b_{2}}\left(x-k_{1} t\right)+C_{1}\right)} .
$$

The analytical solutions (38) and (39) are provided by the conditions (18), (20), and (37) by taking $l=1$. The analytical solutions of Equation (2) are also reported by taking $l=2$.

Family-2: $\left(z_{3}=0\right)$

Set-I: 


$$
\begin{gathered}
z_{0}=b^{2} z_{2}, z_{1}=2 b z_{2}, A_{1}=0, f_{l}=-g_{l}-h_{l}, \\
c_{l}=-\frac{4 d_{l} a_{l}^{2}-\delta_{l} a_{l}^{2}-a_{l}^{2} \zeta_{l}+2 \epsilon_{l} a_{l} b_{l}+2 \eta_{l} a_{l} b_{l}+2 a_{l} b_{l} v_{l}+2 a_{l} b_{l} \theta_{l}}{4 b_{l}^{2}} .
\end{gathered}
$$

As a result, the exact solutions of Equation (1) are retrieved as

$$
q(x, t)=\left\{A_{0}-A_{-1} z_{2}\left(C_{1}+k_{1} t-x\right)\right\} e^{i\left\{\left(k_{2}+\frac{a_{1}}{4 b_{1}} k_{1}\right) t-\frac{a_{1}}{4 b_{1}} x+C_{1}\right\}},
$$

and

$$
r(x, t)=\left\{A_{0}-A_{-1} z_{2}\left(C_{1}+k_{1} t-x\right)\right\} e^{i\left(k_{2} t-\frac{a_{2}}{4 b_{2}}\left(x-k_{1} t\right)+C_{1}\right) .}
$$

The explicit solutions (41) and (42) emerged from the conditions (18), (20), and (40) by taking $l=1$. The solutions of Equation (2) are also given by taking $l=2$.

Set-II:

$$
\begin{gathered}
z_{0}=\frac{z_{2}\left(A_{1} b+A_{0}\right)^{2}}{A_{1}^{2}}, z_{1}=\frac{2 z_{2}\left(A_{1} b+A_{0}\right)}{A_{1}}, A_{-1}=0, f_{l}=\frac{\left(-g_{l}-h_{l}\right) A_{1}^{4}+24 b_{l} z_{2}^{4}}{A_{1}^{4}} \\
c_{l}=\frac{\left\{\left(\zeta_{l}+\delta_{l}\right) a_{l}^{2}-2 b_{l}\left(\theta_{l}+\epsilon_{l}+\eta_{l}+v_{l}\right) a_{l}-4 d_{l} b_{l}^{2}\right\} A_{1}^{2}-3 a_{l}^{2} b_{l} z_{2}^{2}}{4 A_{1}^{2} b_{l}^{2}}
\end{gathered}
$$

Therefore, the analytical solutions of Equation (1) are revealed as

$$
q(x, t)=\left(-\frac{A_{1}}{z_{2}\left(C_{2}+\left(x-k_{1} t\right)\right)}\right) e^{i\left\{\left(k_{2}+\frac{a_{1}}{4 b_{1}} k_{1}\right) t-\frac{a_{1}}{4 b_{1}} x+C_{1}\right\}},
$$

and

$$
r(x, t)=\left(-\frac{A_{1}}{z_{2}\left(C_{2}+\left(x-k_{1} t\right)\right)}\right) e^{i\left\{\left(k_{2}+\frac{a_{1}}{4 b_{1}} k_{1}\right) t-\frac{a_{1}}{4 b_{1}} x+C_{1}\right\}} .
$$

The exact solutions (44) and (45) are considered with the parameters (18), (20), and (43) by taking $l=1$. The explicit solutions of Equation (2) are also recovered by taking $l=2$.

Set-III:

$$
\begin{gathered}
z_{0}=z_{2} b^{2}-\frac{3 a_{l}{ }^{2}}{64 b_{l}{ }^{2} z_{2}}, z_{1}=2 b z_{2}, A_{0}=A_{1}=0, f_{l}=-g_{l}-h_{l}+\frac{243 a_{l}{ }^{8}}{2097152 b_{l}{ }^{7} z_{2}{ }^{4} A_{-1}{ }^{4}}, \\
c_{l}=\frac{\left(\zeta_{l}+\delta_{l}\right) a_{l}{ }^{2}-2 b_{l}\left(\theta_{l}+\epsilon_{l}+v_{l}+\eta_{l}\right) a_{l}-4 d_{l} b_{l}{ }^{2}}{4 b_{l}{ }^{2}}+\frac{81 a_{l}{ }^{6}}{32768 b_{l} z_{2}{ }^{2} A_{-1}{ }^{2}} .
\end{gathered}
$$

Thus, CQ singular optical solitons with Equation (1) are considered as

$$
q(x, t)=-\frac{8 A_{-1} b_{1} z_{2} \sqrt{3}}{3 a_{1}} \operatorname{coth}\left(\frac{a_{1}\left(C_{2}+\left(x-k_{1} t\right)\right) \sqrt{3}}{8 b_{1}}\right) e^{i\left\{\left(k_{2}+\frac{a_{1}}{4 b_{1}} k_{1}\right) t-\frac{a_{1}}{4 b_{1}} x+C_{1}\right\}},
$$

and

$$
r(x, t)=-\frac{8 A_{-1} b_{1} z_{2} \sqrt{3}}{3 a_{1}} \operatorname{coth}\left(\frac{a_{1}\left(C_{2}+\left(x-k_{1} t\right)\right) \sqrt{3}}{8 b_{1}}\right) e^{i\left\{\left(k_{2}+\frac{a_{1}}{4 b_{1}} k_{1}\right) t-\frac{a_{1}}{4 b_{1}} x+C_{1}\right\}} .
$$

The CQ singular optical solitons (47) and (48) are retrieved with the help of the parameter constraints (18), (20), and (46) by taking $l=1$. CQ singular optical solitons with Equation (2) are also yielded by taking $l=2$.

Set-IV:

$$
\begin{gathered}
z_{0}=\frac{z_{2} A_{0}{ }^{2}}{A_{1}{ }^{2}}-\frac{3 a_{l}^{2}}{64 b_{l}{ }^{2} z_{2}}, z_{1}=\frac{2 z_{2} A_{0}}{A_{1}}, f_{l}=-g_{l}-h_{l}+\frac{24 b_{l} z_{2}{ }^{4}}{A_{1}{ }^{4}}, A_{-1}=b=0, \\
c_{1}=\frac{\left(\left(2 \delta_{l}+2 \zeta_{l}\right) a_{l}{ }^{2}-4 b_{l}\left(\epsilon_{l}+\theta_{l}+v_{l}+\eta_{l}\right) a_{l}-8 d_{l} a_{l}{ }^{2}\right) A_{1}{ }^{2}+9 a_{l}{ }^{2} b_{l} z_{2}^{2}}{8 A_{1}{ }^{2} b_{l}{ }^{2}}
\end{gathered}
$$

Hence, CQ dark optical solitons with Equation (1) are structured as

$$
\begin{aligned}
q(x, t)=-\frac{A_{1}}{8 b_{1} z_{2}} & {\left[\tanh \left(\frac{a_{1}\left(C_{2}+x-k_{1} t\right) \sqrt{3}}{8 b_{1}}\right) \sqrt{3} a_{1}-8 b b_{1} z_{2}\right] } \\
& \times e^{i\left\{\left(k_{2}+\frac{a_{1}}{4 b_{1}} k_{1}\right) t-\frac{a_{1}}{4 b_{1}} x+C_{1}\right\}}
\end{aligned}
$$

and

$$
\begin{aligned}
r(x, t)=-\frac{A_{1}}{8 b_{1} z_{2}} & {\left[\tanh \left(\frac{a_{1}\left(C_{2}+x-k_{1} t\right) \sqrt{3}}{8 b_{1}}\right) \sqrt{3} a_{1}-8 b b_{1} z_{2}\right] } \\
& \times e^{i\left\{\left(k_{2}+\frac{a_{1}}{4 b_{1}} k_{1}\right) t-\frac{a_{1}}{4 b_{1}} x+C_{1}\right\}} .
\end{aligned}
$$


The CQ dark optical solitons (50) and (51) are obtained by the aid of conditions (18), (20) and (49) by taking $l=1$. CQ dark optical solitons with Equation (2) are also given by taking $l=2$. Equations (50) and (51) stand for vector dark solitons that come from the reduction in the optical field intensity. These solitons are also less stable compared to scalar dark solitons.

Family-3: $\left(z_{1}=z_{2}=z_{3}=0\right)$

Set-I:

$$
\begin{gathered}
z_{1}=0, z_{2}=0, z_{3}=0, A_{-1}=0, A_{0}=A_{1}=\operatorname{arbitrary}, f_{l}=-g_{l}-h_{l}, \\
c_{l}=-\frac{-\delta_{l} a_{l}{ }^{2}-a_{l}{ }^{2} \zeta_{l}+2 \epsilon_{l} a_{l} b_{l}+2 \eta_{l} a_{l} b_{l}+2 a_{l} b_{l} v_{l}+2 a_{l} b_{l} \theta_{l}+4 b_{l} b_{l}{ }^{2}}{4 b_{l}{ }^{2}} .
\end{gathered}
$$

Consequently, the analytical solutions of Equation (1) are indicated below:

$$
q(x, t)=\left\{A_{0}+A_{1}\left(z_{0}\left(x-k_{1} t\right)+C_{2}+b\right)\right\} e^{i\left\{\left(k_{2}+\frac{a_{1}}{4 b_{1}} k_{1}\right) t-\frac{a_{1}}{4 b_{1}} x+C_{1}\right\}},
$$

and

$$
r(x, t)=\left\{A_{0}+A_{1}\left(z_{0}\left(x-k_{1} t\right)+C_{2}+b\right)\right\} e^{i\left\{\left(k_{2}+\frac{a_{1}}{4 b_{1}} k_{1}\right) t-\frac{a_{1}}{4 b_{1}} x+C_{1}\right\}} .
$$

The exact solutions (53) and (54) are considered with the parameters (18), (20), and (52) by taking $l=1$. The explicit solutions of Equation (2) are also recovered by taking $l=2$.

Family-4: $\left(z_{0}=z_{3}=0\right)$

Set-I:

$$
\begin{gathered}
z_{1}=2 b z_{2}, A_{-1}=A_{0}=0, f_{l}=\frac{\left(-g_{l}-h_{l}\right) A_{1}{ }^{4}+24 b_{l} z_{2}{ }^{4}}{A_{1}{ }^{4}}, a_{l}=\frac{8 z_{2} b b_{l}}{\sqrt{3}}, \\
c_{l}=\frac{4 A_{1}{ }^{2} b z_{2}\left(\eta_{l}+v_{l}+\theta_{l}+\epsilon_{l}\right) \sqrt{3}+\left(16 b^{2}\left(\zeta_{l}+\delta_{l}\right) z_{2}{ }^{2}-3 d_{l}\right) A_{1}{ }^{2}+72 b_{l} b^{2} z_{2}{ }^{4}}{3 A_{1}{ }^{2}} .
\end{gathered}
$$

As a result, CQ straddled optical solitons with Equation (1) are retrieved:

$$
\begin{aligned}
q(x, t)=A_{1}[b+ & \left.\frac{2 b}{-1+2 C_{2} b\left\{\cosh \left(2 b z_{2}\left(x-k_{1} t\right)\right)-\sinh \left(2 b z_{2}\left(x-k_{1} t\right)\right)\right\}}\right] \\
& \times e^{i\left\{\left(k_{2}+\frac{a_{1}}{4 b_{1}} k_{1}\right) t-\frac{a_{1}}{4 b_{1}} x+C_{1}\right\}},
\end{aligned}
$$

and

$$
\begin{aligned}
r(x, t)=A_{1}[b+ & \left.\frac{2 b}{-1+2 C_{2} b\left\{\cosh \left(2 b z_{2}\left(x-k_{1} t\right)\right)-\sinh \left(2 b z_{2}\left(x-k_{1} t\right)\right)\right\}}\right] \\
& \times e^{i\left\{\left(k_{2}+\frac{a_{1}}{4 b_{1}} k_{1}\right) t-\frac{a_{1}}{4 b_{1}} x+C_{1}\right\}} .
\end{aligned}
$$

The CQ straddled optical solitons (56) and (57) are retrieved with the help of the parameter constraints (18), (20), and (55) by taking $l=1$. CQ straddled optical solitons with Equation (2) are also yielded by taking $l=2$.

\section{Conclusions}

The current work derived CQ optical solitons for the perturbed LPD model with differential group delay. The results are confined to dark, singular, and straddled solitons. Lie symmetry analysis yielded the ordinary differential equations that were integrated using Kudryashov's scheme and the improved F-expansion procedure. These two approaches have visible shortcomings. They fail to recover the much-needed bright solitons, which are essential for handling soliton-propagation dynamics across intercontinental distances. Thus, in future additional integration, algorithms are to be implemented to obtain bright solitons for the governing model, which would enable one to paint a complete picture of the governing model. Moreover, the results of this work lead to additional studies, such as addressing the model with the aid of numerical schemes such as the variational iteration approach and Laplace-Adomian decomposition scheme. These results will soon be visible.

Author Contributions: Conceptualization, S.K. and D.H.A.-B.; methodology, H.M.A. and D.A.M.; software, A.B.; writing—original draft preparation, Y.Y. and S.M.; writing-review and editing, L.M. and Y.Y. All authors have read and agreed to the published version of the manuscript.

Funding: This research received no external funding.

Institutional Review Board Statement: Not applicable.

Informed Consent Statement: Not applicable.

Data Availability Statement: All data generated or analyzed during this study are included in this manuscript. 
Acknowledgments: The authors thank the anonymous referees whose comments helped to improve the paper.

Conflicts of Interest: The authors declare no conflict of interest.

\section{References}

1. Ekici, M. Optical solitons in birefringent fibers for Lakshmanan-Porsezian-Daniel model by extended Jacobi's elliptic function expansion scheme. Optik 2018, 172, 651-656. [CrossRef]

2. Kudryashov, N.A. The Lakshmanan-Porsezian-Daniel model with arbitrary refractive index and its solution. Optik 2021, 241, 167043. [CrossRef]

3. Serkin, V.N.; Belyaeva, T.L. Optimal control for soliton breathers of the Lakshmanan-Porsezian-Daniel, Hirota, and cmKdV models. Optik 2018, 175, 17-27. [CrossRef]

4. Xin, H. Optical envelope patterns in nonlinear media modeled by the Lakshmanan-Porsezian-Daniel equation. Optik 2021, 227, 165839. [CrossRef]

5. Kumar, S.; Biswas, A.; Zhou, Q.; Yildirim, Y.; Alshehri, H.M.; Belic, M.R. Straddled optical solitons for cubic-quartic LakshmananPorsezian-Daniel model by Lie symmetry. Phys. Lett. A 2021, 417, 127706. [CrossRef]

6. Vega-Guzman, J.; Biswas, A.; Kara, A.H.; Mahmood, M.F.; Ekici, M.; Alshehri, H.M.; Belic, M.R. Cubic-quartic optical soliton perturbation and conservation laws with Lakshmanan-Porsezian-Daniel model: Undetermined coefficients. J. Nonlinear Opt. Phys. Mater. 2021, 30, 2150007. [CrossRef]

7. Yildirim, Y.; Topkara, E.; Biswas, A.; Triki, H.; Ekici, M.; Guggilla, P.; Khan, S.; Belic, M.R. Cubic-quartic optical soliton perturbation with Lakshmanan-Porsezian-Daniel model by sine-Gordon equation approach. J. Opt. 2021, 50, 322-329. [CrossRef]

8. Bulut, H.; Pandir, Y.; Demiray, S.T. Exact solutions of nonlinear Schrödinger's equation with dual power-law nonlinearity by extended trial equation method. Waves Random Complex Media 2014, 24, 439-451. [CrossRef]

9. Dan, J.; Sain, S.; Ghose-Choudhury, A.; Garai, S. Solitary wave solutions of nonlinear PDEs using Kudryashov's R function method. J. Mod. Opt. 2020, 67, 1499-1507. [CrossRef]

10. Guo, Q.; Liu, J. New exact solutions to the nonlinear Schrödinger equation with variable coefficients. Results Phys. 2020, 16, 102857. [CrossRef]

11. Islam, M.S.; Khan, K.; Arnous, A.H. Generalized Kudryashov method for solving some (3+1)-dimensional nonlinear evolution equations. New Trend Math. Sci. 2015, 3, 46-57.

12. Kudryashov, N.A. First integrals and general solution of the traveling wave reduction for Schrödinger equation with anti-cubic nonlinearity. Optik 2019, 185, 665-671. [CrossRef]

13. Wazwaz, A.M.; El-Tantawy, S.A. Optical Gaussons for nonlinear logarithmic Schrödinger equations via the variational iteration method. Optik 2019, 180, 414-418. [CrossRef]

14. Bluman, G.; Stephen, A. Symmetry and Integration Methods for Differential Equations; Springer: New York, NY, USA, 2008.

15. Olver, P.J. Applications of Lie Groups to Differential Equations; Springer: New York, NY, USA, 2000.

16. Son, N.K.; Thieu, N.N.; Yen, N.D. On the solution existence for prox-regular perturbed sweeping processes. J. Nonlinear Var. Anal. 2021, 5, 851-863.

17. Halik, A. Dynamics in a two species Lotka-Volterra cooperative system with the Crowley-Martin functional response. J. Nonlinear Funct. Anal. 2021, 2021, 36. 